\title{
LA PÉNÉTRATION DU HOTU, CHONDROSTOMA NASUS NASUS (POISSON CYPRINIDÉ), DANS LE RÉSEAU HYDROGRAPHIQUE FRANÇAIS ET SES CONSÉQUENCES.
}

\author{
A. NELVA \\ Université de Savoie, CISM, Laboratoire d'Ecologie des Vertébrés, \\ 73376 Le Bourget du Lac Cedex, France.
}

\section{RÉSUMÉ}

La pénétration du Hotu dans le réseau hydrographique français à la fin du siècle dernier (vers 1860) s'est faite de façon spontanée, à partir du Rhin, par les nouveaux canaux du NordEst. En moins d'une quarantaine d'années, l'espèce s'est étendue au maximum des possibilités dans le bassin de la Seine, puis ceux de la Loire et du Rhône.

Grâce à son adaptation buccale très spécialisée, l'espèce est originale par sa niche alimentaire de périlithophage exclusif, à régime principalement diatomivore. C'est aussi une espèce très grégaire, dont la structure en bancs est liée à la croissance et à l'âge, et qui effectue des migrations saisonnières indispensables à sa survie : elle occupe les moyens et grands cours d'eau, mais remonte dans certains petits affluents au moment de la reproduction, affectionnant les faciès lotiques.

Depuis sa pénétration, et en raison de la réussite de sa naturalisation, l'espèce a été considérée à tort comme nuisible pour le frai de certaines espèces autochtones, en particulier de l'Ombre commun. Des pêches de destruction ont alors été autorisées pendant plusieurs dizaines d'années sur les sites de reproduction communs aux deux espèces, jusqu'à ce que soit récemment démontrée leur inutilité. En réalité, le Hotu n'est concurrentiel pour aucune espèce, pas même pour l'autre Chondrostome autochtone, le Toxostome.

Sensible aux pollutions, mais encore davantage aux aménagements récents qui dégradent ou détruisent ses habitats, l'espèce est en nette régression depuis quelques années. Cette régression est d'autant plus regrettable que le Hotu présente des intérêts écologique (rôle épurateur et bioindicateur) et halieutique certains.

Mots-clés : Chondrostoma nasus (Poisson, Cyprinidae), extension biogéographique, niche écologique, régression actuelle, France, Bassin du Rhône.

\section{THE PENETRATION OF THE NASE, CHONDROSTOMA NASUS NASUS (PISCES, CYPRINIDAE), IN THE FRENCH HYDROGRAPHIC NETWORK AND ITS CONSEQUENCES.}

\begin{abstract}
The expansion of nase in French rivers occurred spontaneously, starting about 1860 from the Rhine and passing through the new canal system of Northeastern France. This species reached its maximum extent in the Seine, then in the Upper Loire and Rhone basins within less than forty years.
\end{abstract}


The species has an exclusively perilithophagic, especially diatomivorous, diet, owing to its very specialised mouth. It is a gregarious species, whose schooling is governed by size and age. Complex movements or migrations linked with spawning and growth are performed between the main channel and side arms or tributaries, with a progressive preference for the main channel of rivers as age increases.

The species has been considered harmful because of the success of its naturalisation and because it sometimes invaded some portions of rivers in which it was suspected of feeding on grayling eggs. Extermination fishing was authorised during tens years on some spawning grounds common to nase and grayling, but its uselessness has now been proved. In fact, nase does not compete with any other species, the native Southwest European nase, Chondrostoma toxostoma, included.

Nase appears quite sensitive to pollution and even more to the destruction of its habitat. Its populations have been declining markedly for some years. Nevertheless it is a species of great ecological and fishing interest.

Key-words : Chondrostoma nasus (Pisces, Cyprinidae), biogeographic expansion, ecological niche, present decline, France, Rhone Basin.

\section{INTRODUCTION}

Le genre Chondrostoma, auquel appartient le Hotu, n'est représenté en France que par les deux espèces rhéophiles : C. toxostoma (Toxostome) et C. nasus (Hotu), sur les 23 à 25 espèces ou sous-espèces reconnues (NELVA et al., 1988).

La première est autochtone dans le sud de la France (sous-espèce $C$. $t$. toxostoma des bassins du Rhône, de la Garonne et de l'Adour), ainsi que dans le Nord-Est de la péninsule ibérique (sous-espèce C. $t$. miegi). Elle ne semble avoir bénéficié des canaux du centre de la France qu'assez récemment, dans les années 1930-1940, pour passer dans le bassin de la Loire moyenne.

La deuxième, sous-espèce $C$. $n$. nasus, originaire du Danube, de l'Oder, de la Vistule puis du Rhin, s'est spontanément introduite dans le réseau hydrographique français dès le milieu du 19ème siècle par les canaux de l'est puis du centre de la France, et a étendu son aire de répartition au maximum de ses possibilités géographiques en une quarantaine d'années. Beaucoup plus typique que le Toxostome par son comportement alimentaire et sa grégarité, le Hotu présente également une plus grande fécondité, une croissance plus rapide et une taille maximale double (jusqu'à $51 \mathrm{~cm}$, rarement plus, contre moins de $28 \mathrm{~cm}$ pour le Toxostome). L'analyse des principaux caractères méristiques et plastiques des populations du Rhône (NELVA, 1985) montre que celles-ci appartiennent à la même sous-espèce originelle d'Europe centrale, et que les deux formes elata et elongata n'y sont pas différenciées comme dans le Danube. Par ailleurs, un polymorphisme enzymatique, élevé mais remarquablement commun aux populations même éloignées de ce fleuve (GOLLMANN, 1995), indique un haut degré de liens génétiques, c'est-à-dire peu de diversité génétique entre ces populations. II en serait de même pour les populations du Rhône (analyses en cours).

Après avoir rappelé l'historique de sa pénétration et sa répartition actuelle, nous montrons d'abord ce qui fait l'originalité de cette espèce et qui explique la réussite de sa naturalisation rapide dans les zones à Ombre et à Barbeau de nos cours d'eau. Puis, nous indiquons quelques-unes des conséquences de cette introduction sur le Toxostome et surtout sur l'Ombre commun, avec lesquels il cohabite fréquemment. Nous donnons ensuite les perspectives de cette espèce en voie de régression relativement sensible aux dégradations de la qualité de l'eau et des habitats. Enfin, nous essayons de dresser un bilan de la pénétration du Hotu dans le réseau hydrographique français, en soulignant l'intérêt de cette espèce sur les plans écologique et halieutique. 


\section{RAPPEL DE L'HISTORIQUE DE LA PÉNÉTRATION DU HOTU ET RÉPARTITION ACTUELLE EN FRANCE}
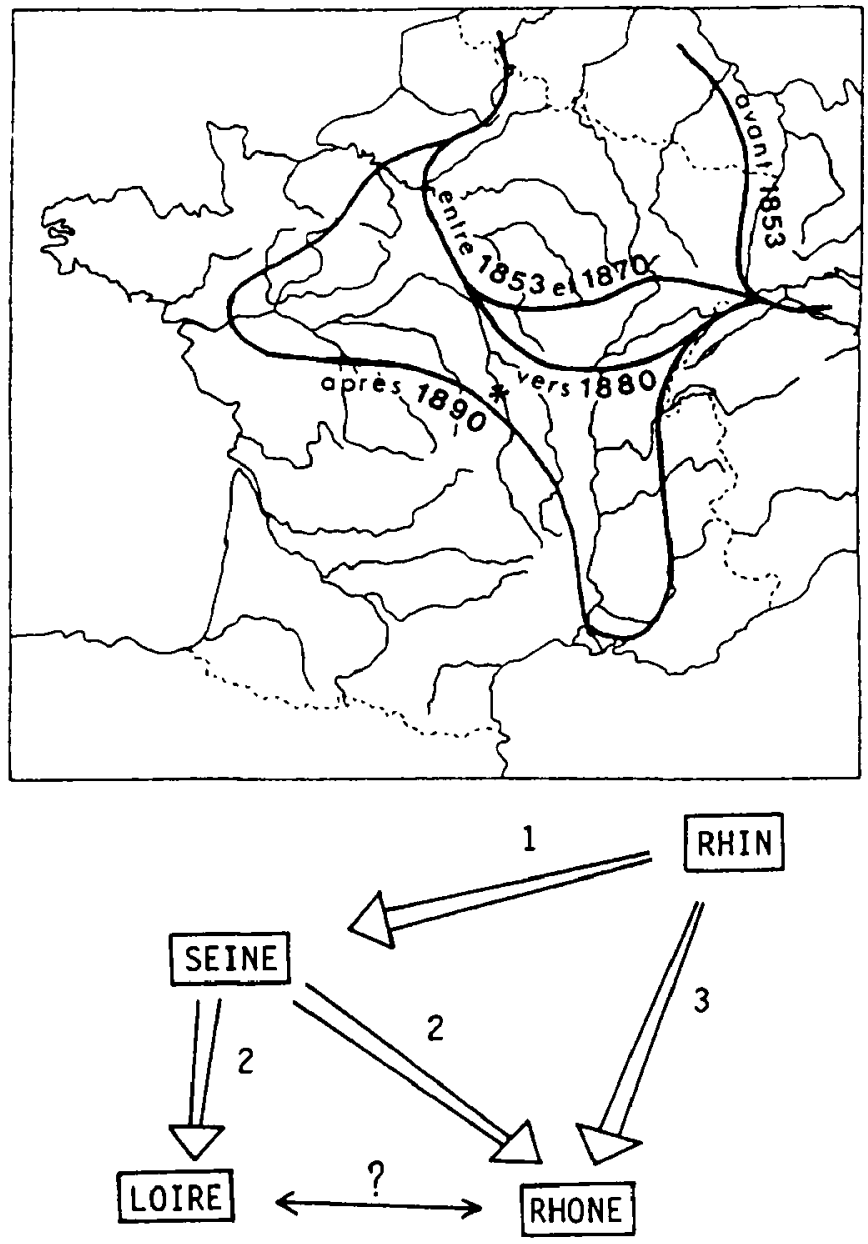

Figure 1

Progression schématique probable du Hotu dans le réseau hydrographique français (* introduction vers 1873).

Figure 1

Scheme of the probable extent of Nase in French rivers ( ${ }^{\star}$ introduction about 1873).

La pénétration du Hotu dans le réseau hydrographique français (NELVA, 1988 a) ne semble avoir commencé qu'après 1853, mais elle s'est réalisée surtout entre 1880 et 1890 de façon involontaire et progressive, mais assez rapide puisqu'en une quarantaine d'années, elle a atteint l'apogée de son extension (Fig. 1). Des introductions directes, signalées dans l'Allier vers 1872-1873 et plus tard dans le Sud-Ouest, ne semblent pas avoir joué un rôle appréciable, d'autant qu'elles ont été souvent le fait de confusions avec plusieurs espèces (Ombre, Blageon, Vandoise, Chevaine ou même Fera). En recoupant les données historiques, dates exactes de mises en service des différents canaux de navigation de l'Est et du Centre de la France d'une part, et signalements de l'espèce dans la littérature scientifique d'autre part, la pénétration du Hotu s'explique en fait par son passage momentané dans ces canaux pour circuler d'un bassin à l'autre. L'extension s'est réalisée, semble-t-il, en trois étapes : 
- d'abord, pénétration à partir du Rhin dans la Seine, probablement par le canal de la Marne au Rhin et la Moselle (en une vingtaine d'années), mais peut-être aussi par la Meuse et l'Escaut (possible en une dizaine d'années seulement), entre 1853 et 1862-1870;

- ensuite, passage de la Seine à la Loire et certainement au Rhône, où il est signalé surtout à partir de 1880 ; l'extension s'est enfin poursuivie jusqu'à la fin du siècle (années 1890).

- l'itinéraire 3 (Fig. 1) n'aurait été emprunté que plus tardivement, et l'espèce aurait mis entre dix et vingt années pour atteindre le Rhône depuis la Seine et la Loire. Cette pénétration, d'origine anthropique, a dû être ralentie par de nombreux obstacles (écluses, chutes d'eau), mais favorisée par la proximité de rivières le long des canaux, car cette espèce rhéophile et relativement exigeante pour la qualité des cours d'eau n'est jamais abondante dans les canaux eux-mêmes. Elle a été limitée par les barrières naturelles séparant les bassins ou interrompant les cours d'eau, par les barrages, et enfin par des barrières écologiques liées aux propres limites de tolérance de l'espèce vis-à-vis du milieu et des pollutions. D'autres espèces également ont su utiliser ces nouvelles voies d'eau pour se propager, par exemple le Sandre, les Brèmes, la Grémille, la Perche-Soleil, le Poisson-Chat, le Black-bass, le Spirlin, la Lotte. La carte de répartition actuelle (NELVA, 1988 a) (Fig. 2) correspond encore à son extension maximale, mais avec des discontinuités de plus en plus marquées.

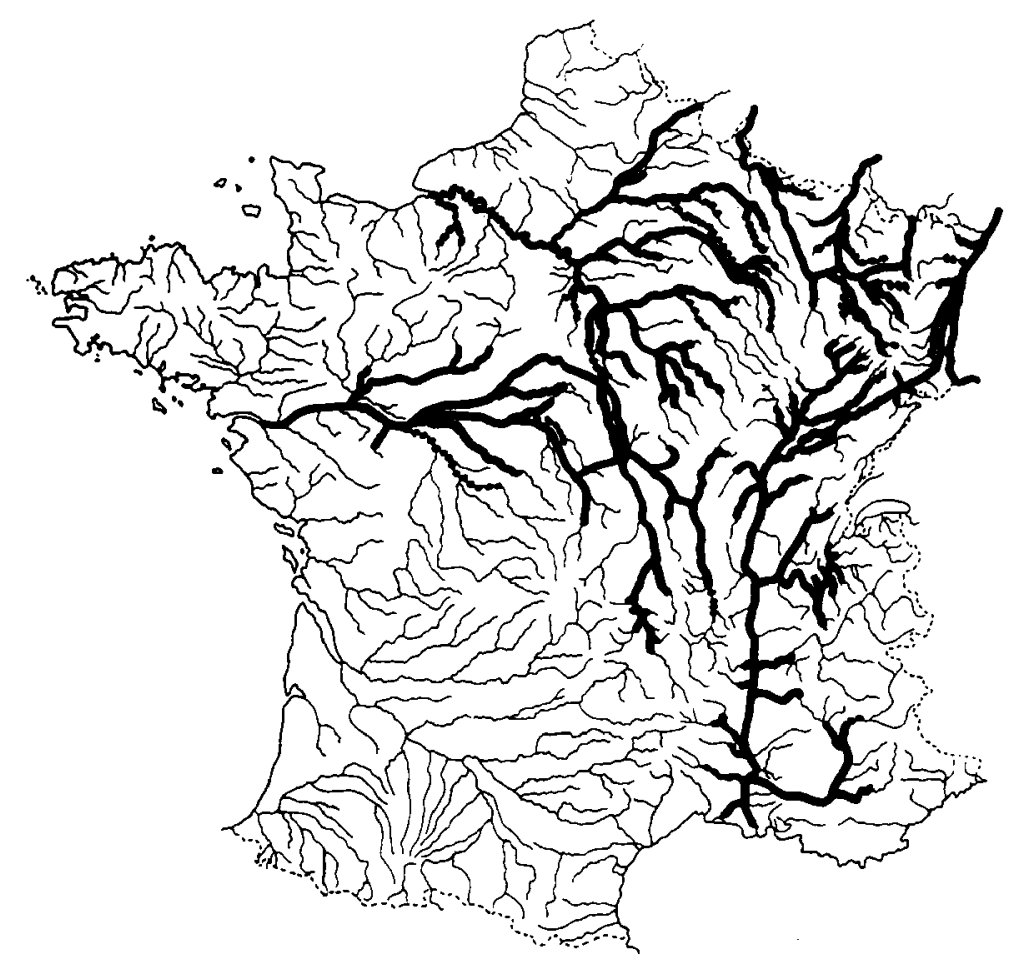

\section{Figure 2}

Biogéographie actuelle du Hotu dans le réseau hydrographique français (traits pleins : présence certaine - pointillés : présence rare ou probable).

Figure 2

Current biogeography of Nase in French rivers (dotted lines : rare or probable occurrence). 


\section{ORIGINALITÉS DE L'ESPÈCE}

1. Niche alimentaire

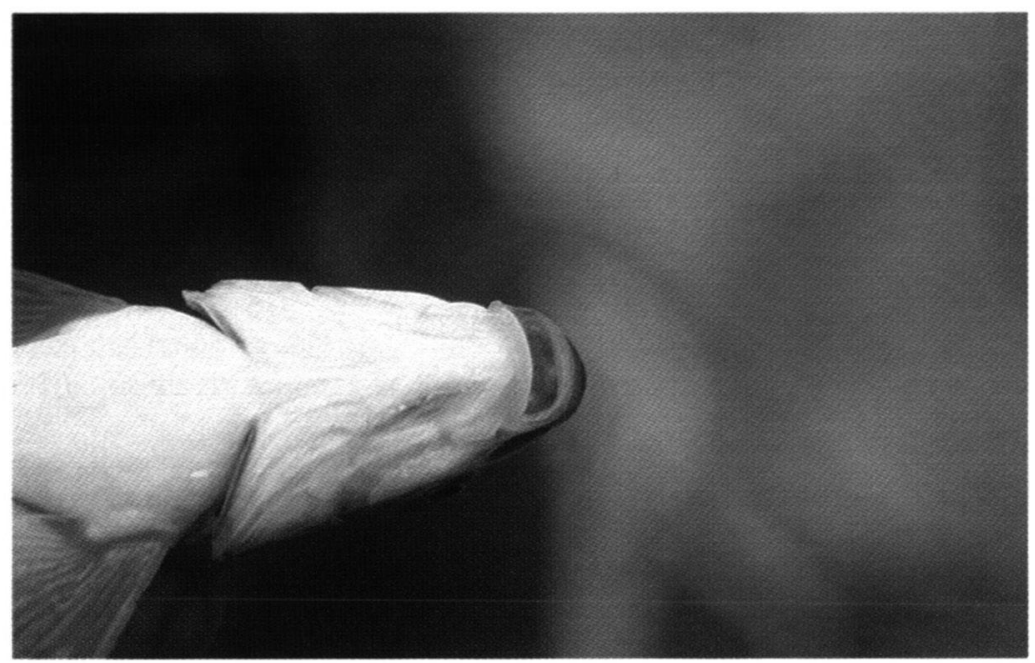

\section{Photo a}

Face ventrale de la bouche du Hotu, montrant la lèvre inférieure cornée.

\section{Photo a}

Ventral face of Nase mouth, showing the horny lower lip.

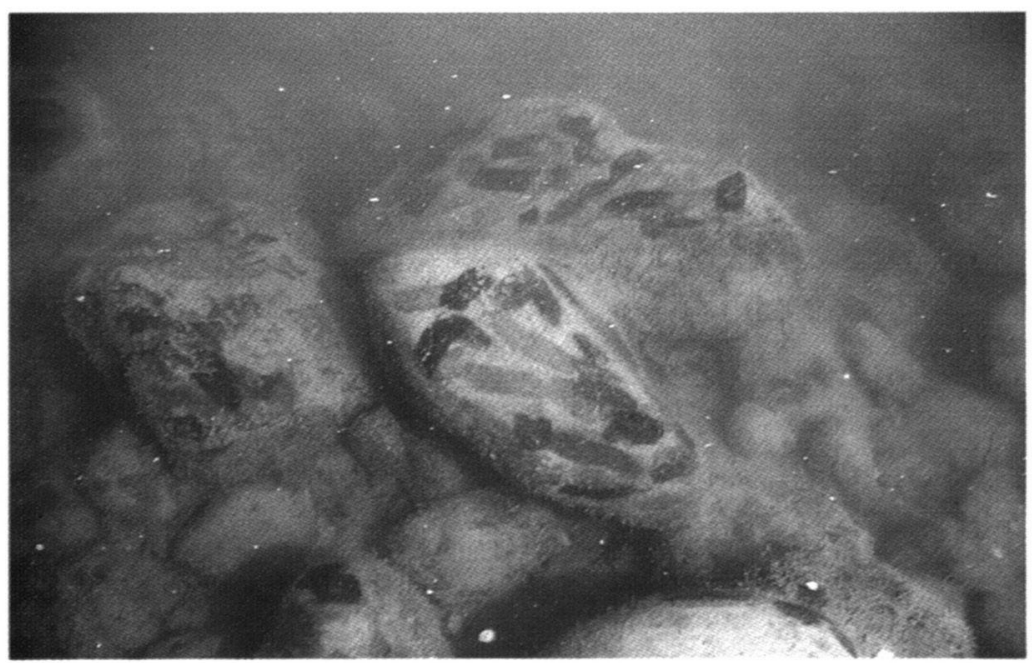

\section{Photo b}

Traces de raclage de Hotus sur le substrat.

\section{Photo b}

Scraping trails of Nases on stones. 
Grâce à une adaptation buccale originale (photo a), le Hotu racle intégralement la couverture biologique composée essentiellement d'algues, surtout de Diatomées, avec un faible pourcentage de micro-organismes liés à cet habitat. II est, de ce fait, le seul périlithophage exclusif de l'ichtyofaune européenne, et l'un des rares herbivores stricts d'eau douce (tab. I). Les juvéniles ont une part animale importante dans leur régime alimentaire, comme pour la plupart des espèces, mais dès qu'ils acquièrent leur lèvre cornée et rectiligne et leur comportement de raclage, vers 5-7 mois, le régime devient diatomivore. La croissance du tube digestif, comme celle des lèvres cornées, est directement dépendante de la taille ou de

\section{Tableau I}

Contenu du tube digestif de Hotus (secteur de Chautagne, Haut-Rhône) : 10 adultes de 369 à 440 $\mathrm{mm}$ (nombre de taxons, en haut) et 30 juvéniles de 12 à $116 \mathrm{~mm}$ (occurrence, en bas).

\section{Table I}

Gut contents of Nases (Chautagne area, upper Rhône) : ten adults 369-440 mm (upper table, taxa number) and thirty juveniles $12-116 \mathrm{~mm}$ (lower table, occurrence).

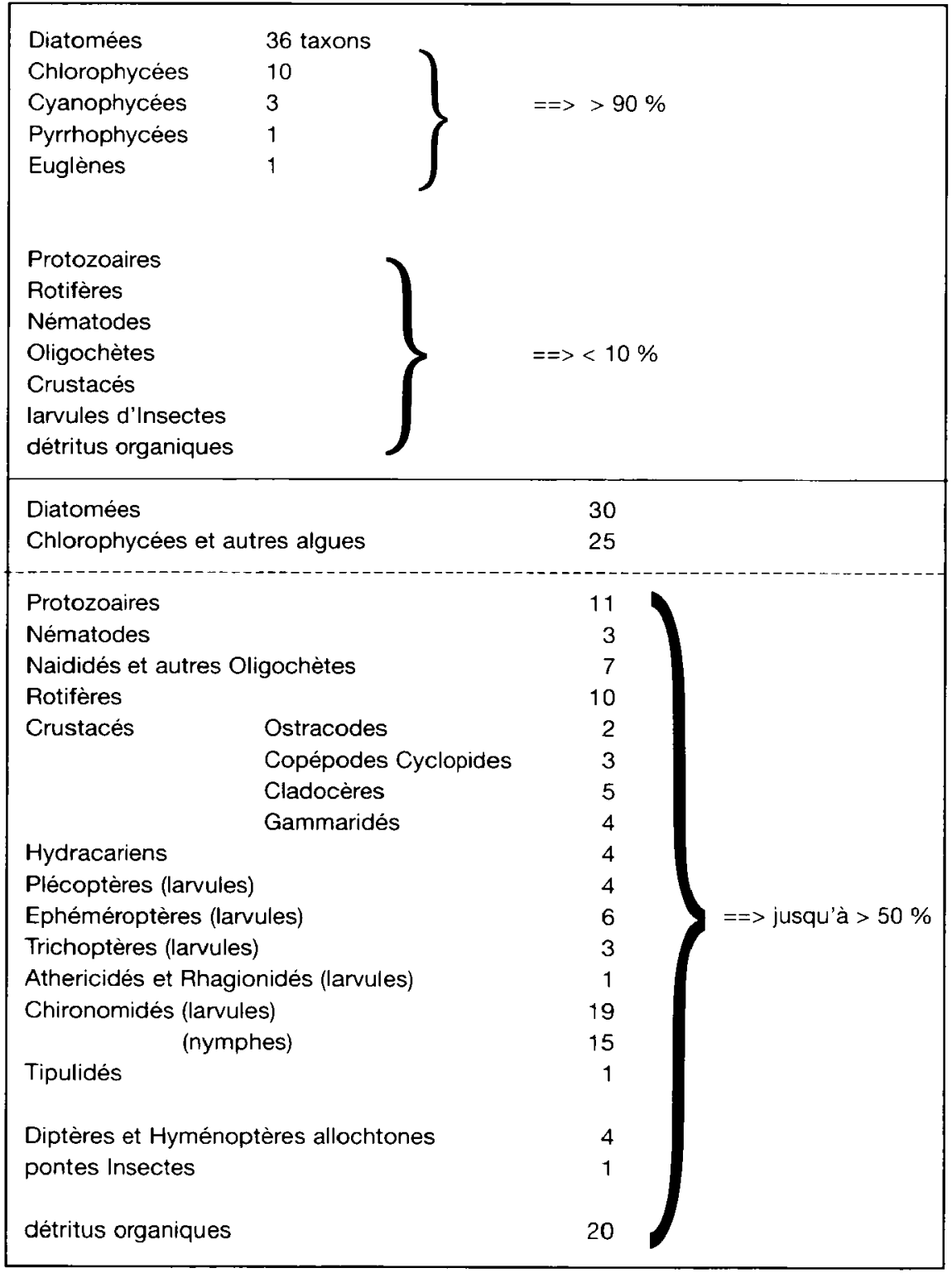


l'âge ; il est long, comme chez tous les herbivores (1,2 à 2 fois la longueur du corps chez des individus du Haut-Rhône, jusqu'à 4 fois chez des individus de grande taille dans le Danube), et ne contient que très peu de parasites intestinaux. Les traces de raclage visibles sur le substrat (photo $b$ ) peuvent être exploitées comme indicateur qualitatif de présence et de détermination des différents groupes de taille, mais pas comme indicateur quantitatif de la structure de taille des populations (FREYHOF, 1995). L'accumulation de lipides dans la chair provient de la dégradation des Diatomées par des complexes enzymatiques, en particulier une laminarinase nécessaire à l'hydrolyse de certains composés algaux. Enfin, la prise alimentaire est essentiellement diurne, inconstante, nulle pendant la reproduction, et n'a été observée qu'entre $4-5^{\circ} \mathrm{C}$ et $23-24^{\circ} \mathrm{C}$ (NELVA, 1985).

\section{Grégarité}

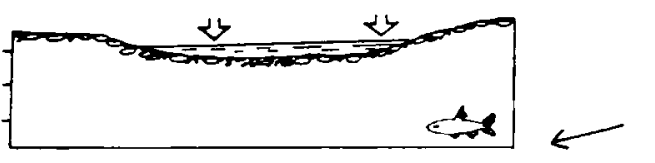

juvéniles $3-7 \mathrm{~cm}, 0+$ a 1 an rive pente faible ou largeur trés étroite profondeur faible $(<50 \mathrm{~cm})$ vitesse du courant nulle et faible

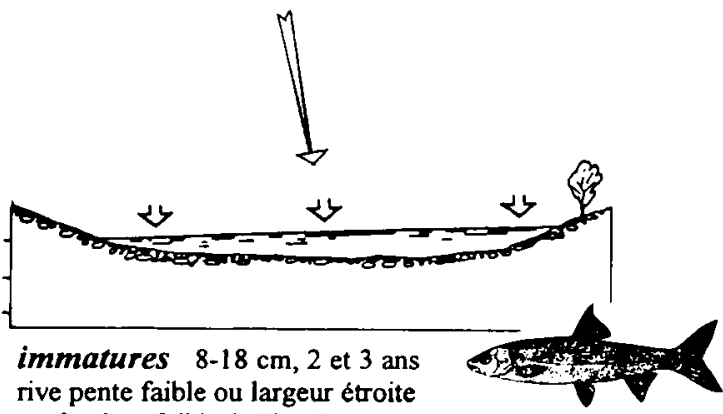
profondeur faible $(<50 \mathrm{~cm})$ vitesse du courant moyenne sites de reproduction

(2-4 semaines en mars ou avril) zones très étroites ou étroites profondeur très faible vitesse du courant moyenne à forte

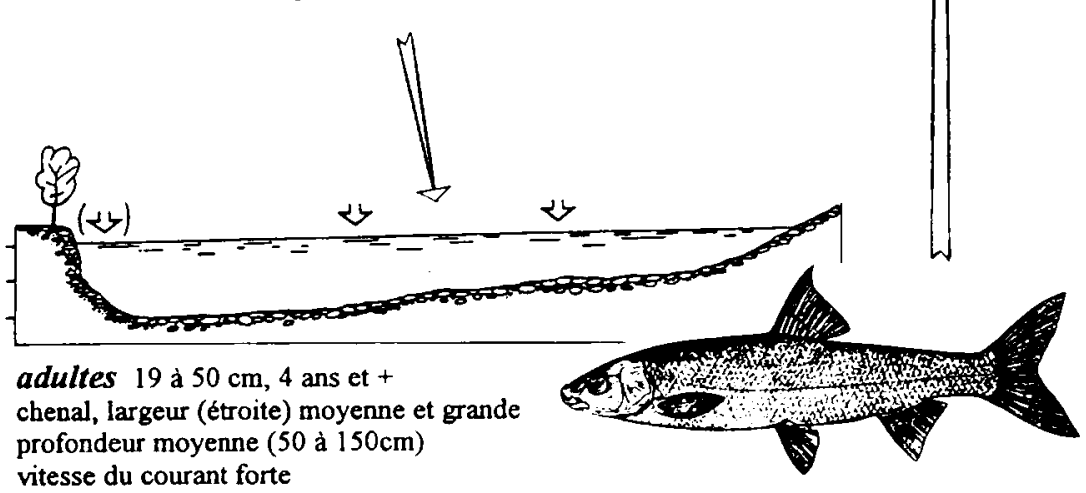

Figure 3

Dynamique spatio-temporelle du Hotu au cours de sa croissance dans le Haut-Rhône à partir d'une description du profil écologique de chaque banc dans une typologie de l'habitat ponctuel par Analyse Factorielle des Correspondances Multiples et Analyse Discriminante.

Figure 3

Spatio-temporal dynamics of Nase growth in the upper Rhône from ecological profiles of three types of schools and from typology of point habitat. 
A partir de la répartition spatio-temporelle des classes de taille centimétriques dans des échantillons des populations du Haut-Rhône, trois types de bancs bien définis par les correspondances taille/âge et l'étude de la croissance ont été identifiés (NELVA, 1989) (Fig. 3) : les juvéniles (jusqu'à un an), les immatures (âgés de 2 et 3 ans), les adultes auxquels se joignent quelques aînés des immatures proches de la maturité (âgés de 4 ans et au-delà). La grégarité est élevée surtout chez les immatures. Les adultes constituent des bancs typiquement monospécifiques. En revanche, juvéniles et immatures forment des bancs plurispécifiques. Les formations en bancs de deuxième et troisième types ne sont pas vraiment liées à l'état de maturité sexuelle, mais plutôt déterminées en fonction de la taille individuelle qui dépend elle-même des conditions écologiques dans les cours d'eau, donc de la croissance : dans un affluent du Danube, en effet, où la croissance est nettement plus lente, la Rokytna (LUSK, 1967), il existe également trois types de bancs, mais le deuxième type contient quelques adultes associés aux immatures, avec des individus jusqu'à 5 et 6 ans et la séparation s'effectue à $24 \mathrm{~cm}$ au lieu de 18-19 cm dans le Rhône.

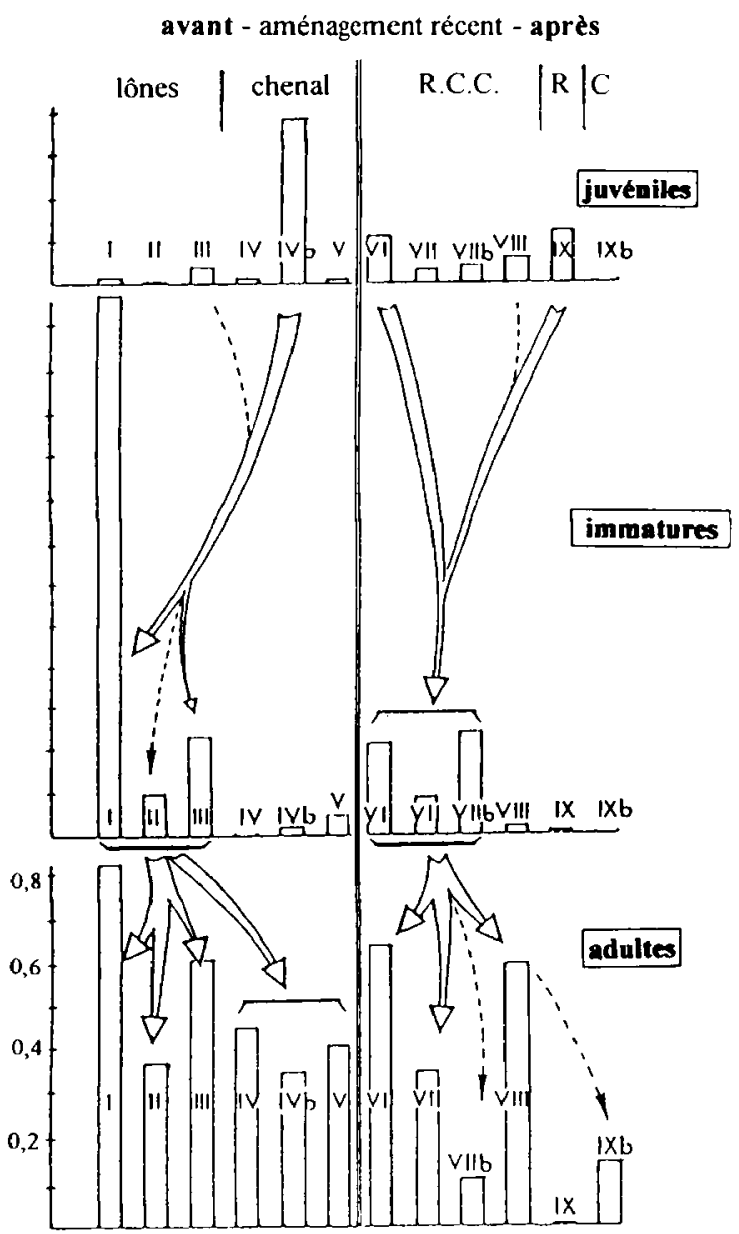

\section{Figure 4}

Evolution de la mésorépartition du Hotu dans les habitats stationnels au cours de sa croissance dans les 12 unités (abscisses) d'une typologie d'habitat stationnel (ordonnées : effectifs par échantillon), après une Analyse Discriminante (échantillons ponctuels regroupés en stations) (RCC : Rhône court-circuités, R : retenues, $\mathrm{C}$ : canaux).

Figure 4

Distribution of Nase according to a station habitat typology during its growing period : number per sample (Y-axis) at the 12 stations units (X-axis) by AD (RCC : by-passed sections, R : reservoirs, $\mathbf{C}$ : channels). 
Cette structure en bancs dans le Haut-Rhône a été analysée à deux échelles d'observation du milieu : I'habitat ponctuel et l'habitat stationnel (NELVA, 1988 b). Au niveau ponctuel (Fig. 3), l'analyse montre un déplacement progressif des bancs d'un type d'habitat à un autre (glissement d'une modalité à l'autre dans plusieurs variables du milieu) et indique une nette tendance à la " chenalisation " des individus au cours de leur croissance et leur installation par étapes successives dans un espace de plus en plus vaste. Ainsi, au cours de sa croissance, le Hotu effectue un cycle d'occupation de l'espace dans une structure en bancs originale.

Au niveau stationnel (Fig. 4), et avant les aménagements récents du fleuve, le Hotu n'occupe également les différentes unités d'habitat définies dans une typologie stationnelle qu'au fur et à mesure de sa croissance, avec des déplacements des bancs entre chenal et lônes. Dans les nouveaux tronçons court-circuités par les aménagements, la réduction des débits semble augmenter sensiblement la diversité des habitats correspondant aux trois types de bancs, assurant ainsi dans cette seule partie résiduelle du fleuve le maintien des populations, mais plus ou moins isolées et déstabilisées par la régulation artificielle des débits. Dans les retenues, où ne sont échantillonnés que des individus de petites tailles, et dans les canaux, où ne peuvent circuler que quelques adultes lorsque le fleuve n'est pas trop éloigné, le manque de diversité des habitats ne permet pas d'assurer la dynamique de croissance décrite, et nécessite des déplacements entre le chenal et les lônes ou affluents.

\section{Fécondité et croissance}

La comparaison de la démographie des populations du Haut-Rhône (NELVA, 1985) avec celle d'autres populations, en particulier des bassins du Danube et du Rhin, montre que la croissance linéaire et pondérale est la plus forte dans des rivières caractéristiques de la "zone à Hotu ", comme la rivière d'Ain (Haut-Rhône) ou l'Ourthe (Meuse), ainsi que la fécondité absolue (femelles de plus grande taille). De plus, la maturité sexuelle y est plus précoce, dès 3 ou 4 ans, contre 5 à 7 ans généralement. Les valeurs sont donc plus élevées que dans beaucoup d'autres populations d'Europe centrale étudiées et qui ont une croissance moins rapide. La plus grande fécondité du Hotu par rapport aux autres Cyprinidés rhéophiles, Chevaine, Vandoise, Barbeau (PHILIPPART, 1977) compense une mortalité initiale très forte dans un environnement variable et imprévisible (espèce à stratégie " $r »)$.

\section{CONSÉQUENCES SUR LES POPULATIONS DU TOXOSTOME ET DE L'OMBRE COMMUN}

Le Toxostome est une espèce dont il est difficile de connaître avec précision l'aire biogéographique antérieure à l'arrivée du Hotu. Mais contrairement à celui-ci, le Toxostome ne constitue jamais des stocks importants. La compétition entre les deux espèces, là où elles cohabitent vraiment, essentiellement dans certains affluents du Bas-Rhône, n'apparaît pas très importante du fait des différences morphologiques (taille, forme de la bouche) et dans la mesure où la ressource alimentaire disponible est suffisamment abondante. Le Hotu est en fait un racleur beaucoup plus strict et efficace à cause de sa lèvre cornée rectiligne et dure : les deux espèces ne doivent sans doute pas occuper exactement la même niche alimentaire. S'il y avait eu d'ailleurs une forte compétition, elle se serait manifestée peu après l'arrivée du Hotu au détriment du Toxostome, démographiquement moins compétitif. Or, il semble que les deux populations aient maintenu une certaine stabilité de leurs aires de répartition, après un recul probablement limité du Toxostome dans les zones les plus propices au Hotu. PARIS (1932) indique que le Toxostome cédait peu à peu la place au Hotu dans la Saône moyenne, en se défendant cependant dans les affluents; SPILLMANN (1961) et d'autres auteurs depuis mentionnent le Toxostome dans la plupart des affluents du Bas-Rhône, tandis que le Hotu vit plutôt dans le fleuve et ne remonte les affluents que sur de courtes distances. Avec les migrations saisonnières des Hotus, et une cohabitation parfois transitoire, cette répartition globale se maintient depuis des années dans les affluents, dont l'Ardèche constitue un exemple type (NELVA, 1985). 
Le Hotu ne porte pas non plus de préjudice notable aux autres espèces autochtones, en particulier vis-à-vis de l'Ombre commun dont il a été très longtemps soupçonné de dévorer les pontes et les larves lors des remontées des reproducteurs, et pour ce fait considéré comme nuisible. En dépit de toute étude sérieuse, le Hotu, peu après son arrivée, a fait l'objet de destructions systématiques au moment des rassemblements sur les frayères. Par exemple dans le Suran, affluent de la rivière d'Ain (Haut-Rhône), les destructions ont commencé dès 1901 pour ne se terminer qu'en 1982, soit pendant 80 ans. Suite aux investigations menées sur ce même site (NELVA, 1985) et aux observations de nombreux auteurs, plusieurs arguments ont conduit à déconsidérer la " nuisibilité " du Hotu :

1- il existe un décalage de 3 semaines environ, en général sans chevauchement, entre le début du frai de l'Ombre (mars) et celui du Hotu (avril) (PHILIPPART, 1977 ; KOKUREWICZ et al., 1980) ;

2- la granulométrie du substrat des pontes est fréquemment différente (graviers pour l'Ombre, cailloux ou galets pour le Hotu); site ;

3- les larves d'Ombre sont enfouies, donc inaccessibles lorsque les Hotus arrivent sur le

4- peu avant et pendant la période de reproduction, les Hotus reproducteurs ne se nourrissent pas (observation de 88 individus dans le Suran et le canal de Miribel ainsi que GYURKO, 1959 et TUFFERY, 1969) ;

5- ils sont racleurs de surface (périlithon), et non suceurs du benthos ;

6- les graviers instabilisés par le courant sont peu propices au développement du périlithon ;

7- inversement, les amas d'oeufs de Hotus constituent un apport alimentaire conséquent pour plusieurs espèces dont l'Ombre commun, le Chevaine, le Goujon, le Barbeau, le Spirlin, la Loche franche, la Vimbe (PENAZ et LUSK, 1965 ; LIBOVARSKY, 1977), ce qui peut entraîner un déclin du Hotu;

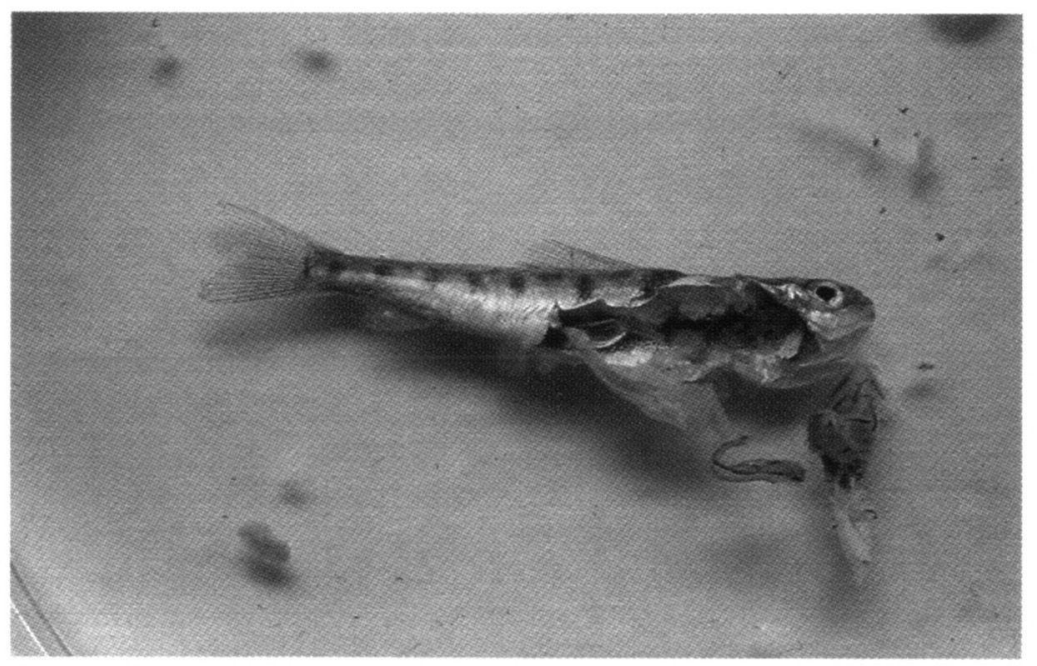

\section{Photo c}

Dissection du tube digestif d'un juvénile d'Ombre commun de $43,5 \mathrm{~mm}$ contenant une larve de Hotu de $13 \mathrm{~mm}$.

\section{Photo c}

Digestive tract dissection of a Grayling juvenile $(43.5 \mathrm{~mm}$ ) holding a Nase larva (13 $\mathrm{mm}$ ). 
8- les larves et juvéniles de Hotus constituent également des proies faciles pour les jeunes Ombres (comportement seulement observé en conditions expérimentales pour le moment mais qui rend ce type de prédation très probable dans le milieu naturel) (photo c), pour les Ombres adultes et pour d'autres prédateurs également (PENAZ et LUSK, LIBOVARSKY, op. cit.) ;

9- depuis la suspension des pêches de destruction des Hotus en 1982, la reproduction de l'Ombre reste aussi satisfaisante qu'auparavant, et le peuplement du cours d'eau aussi diversifié ;

10- en revanche, la pratique des pêches de destruction entraînait un risque évident pour le peuplement du cours d'eau (piétinement intempestif du substrat, notamment des frayères à Ombre où sont enfouies les larves, choc électrique et manipulations dangereuses des poissons, perturbations des biocénoses).

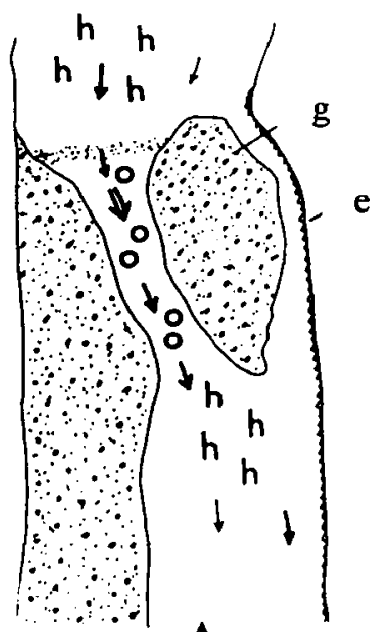

A

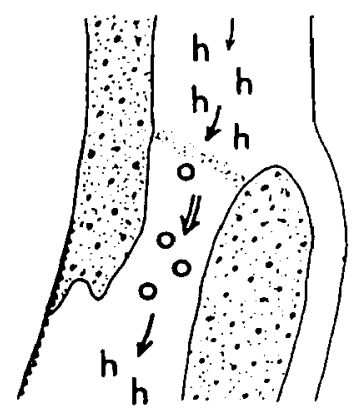

D

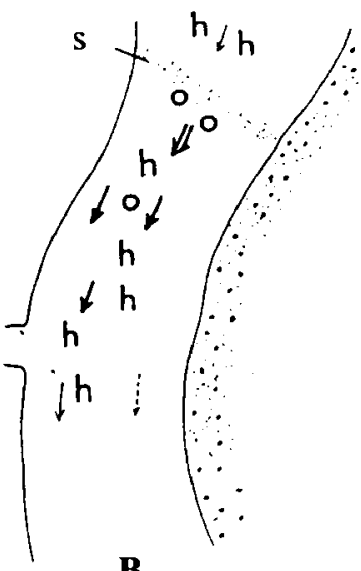

B

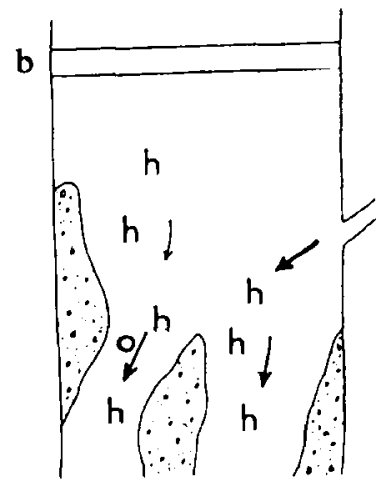

$\mathbf{E}$

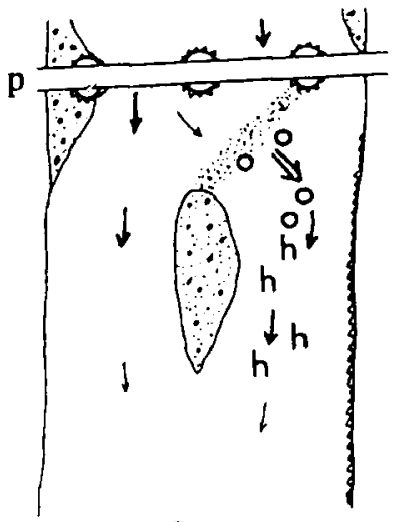

C

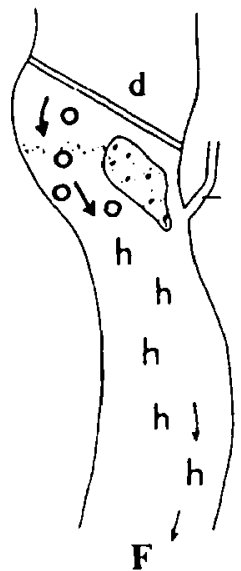

\section{Figure 5}

Quelques cas de microrépartition fréquente du Hotu (h) et de l'Ombre (o) dans des rapides du Rhóne court-circuité de Chautagne (A et B) et de Belley (C et D), du canal de Miribel (E) et du Suran (F). Les flèches indiquent le sens et les vitesses du courant (moyenne, forte, très forte). $b$ : barrage, $d$ : digue, $e$ : enrochements, $g$ : graviers, $p$ : pont, $s$ : seuil.

\section{Figure 5}

Examples of frequently occurring microdistributions of Nase ( $h$ ) and Grayling (o) in by-pass rapids of Chautagne ( $A$ and $B$ ) and Belley $(C$ and $D)$ dams, Miribel channel $(E)$ and Suran river $(F)$. $b$ : dam, d and $e$ : embankment, $g$ : gravels, $p$ : bridge, $s$ : sill. 
Très proches pour les conditions de reproduction (sites, périodes, température de l'eau, vitesse du courant), les deux espèces, Ombre et Hotu, le sont également pour les habitats, dans les faciès les plus courants où le premier capture ses proies et le second racle sa nourriture. La figure 5 montre des exemples de situations fréquentes d'occupation de l'espace dans les zones de cohabitation. Le Hotu est d'autre part sensible à l'élévation de la température de l'eau (NELVA, 1984), comme l'Ombre. C'est donc un Cyprinidé rhéophile exigeant, mais qui sait aussi s'accommoder de conditions moins sévères, comme celles de la zone à Barbeau. Cette tolérance lui a permis de pénétrer dans les canaux du Nord-Est de la France il y a plus d'un siècle. Une "zone à Hotu " chevauchant les deux zones, à Ombre et à Barbeau, et définissant bien les secteurs moyens des grandes rivières et l'aval de leurs affluents, est déjà reconnue en Europe centrale. En pénétrant dans nos cours d'eau, l'espèce a ainsi trouvé des conditions très favorables, principalement une niche alimentaire largement disponible, sans nuire aux espèces autochtones.

\section{RÉGRESSION ACTUELLE DES POPULATIONS DE HOTU}

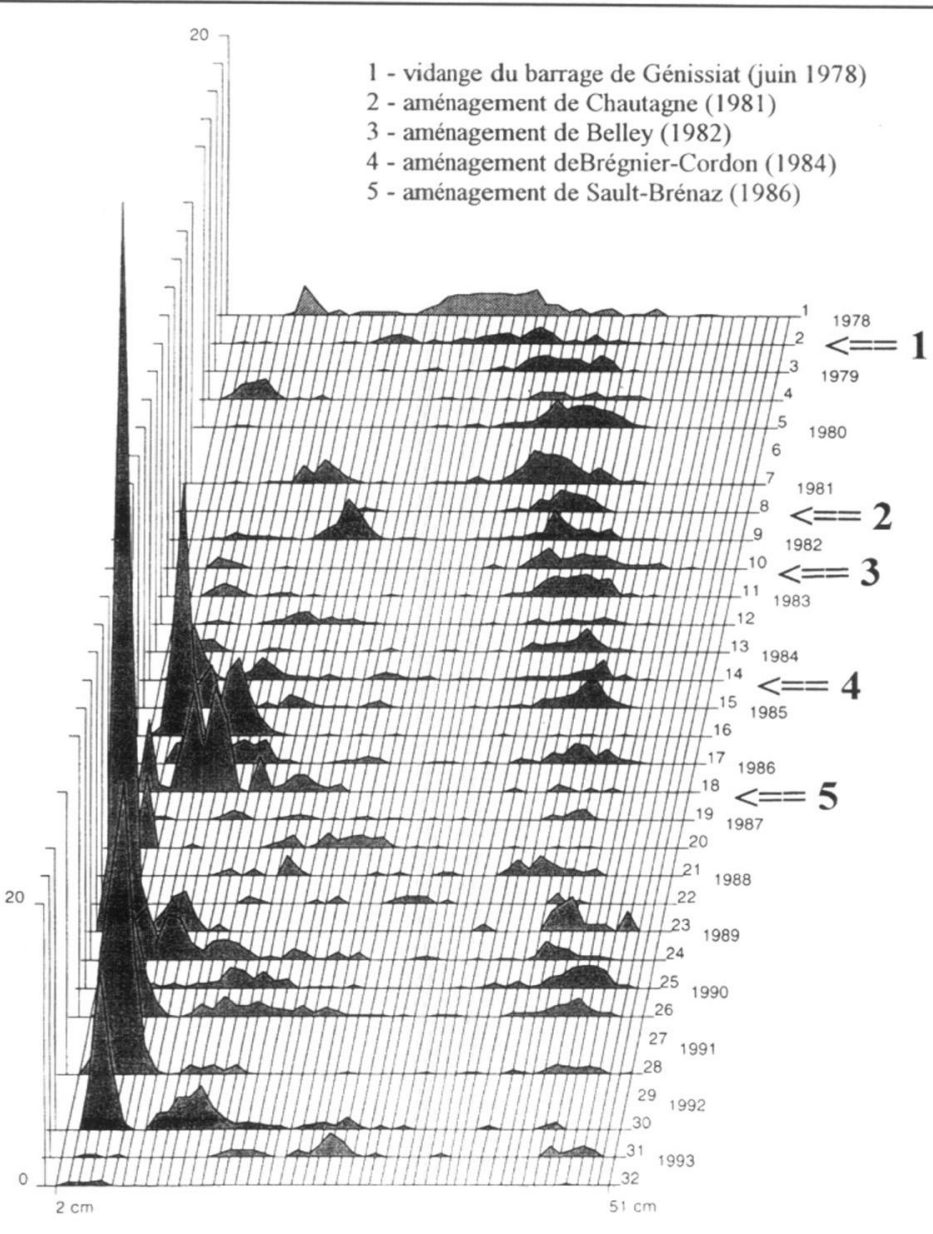

Figure 6

Modifications de la structure en classes de taille des populations de Hotu dans le Haut-Rhône de 1978 à 1993 (classes de taille centimétriques en abscisse, nombre moyen d'individus par effort de pêche en ordonnée) (PERSAT et al., 1994).

Figure 6

Changes in the size-class structure of the populations of Nase in the upper Rhône from 1978 to 1993 (average number of specimens per fishing effort) (PERSAT et al., 1994). 
Sensibles aux pollutions, mais aussi aux divers aménagements des cours d'eau qui dégradent ou font disparaître leurs habitats, les populations régressent ou disparaissent de certaines portions de cours d'eau, en France comme dans l'aire d'origine. Par exemple dans le Haut-Rhône (Fig. 6), malgré une récupération des populations après la vidange catastrophique des barrages réservoirs en 1978, les échantillonnages réguliers de 1978 à 1993 (PERSAT et al., 1994) montrent un déclin progressif des grandes tailles, c'est-à-dire leur vieillissement sans renouvellement, commencé avec l'achèvement du programme d'aménagement du fleuve. Pourtant de fortes cohortes de juvéniles certaines années, donc un recrutement annuel presque normal, subsistent un ou deux ans. Dans ce même secteur, la population du Lac du Bourget ne devrait plus survivre longtemps non plus, car le Hotu ne peut plus y pénétrer depuis le Rhône
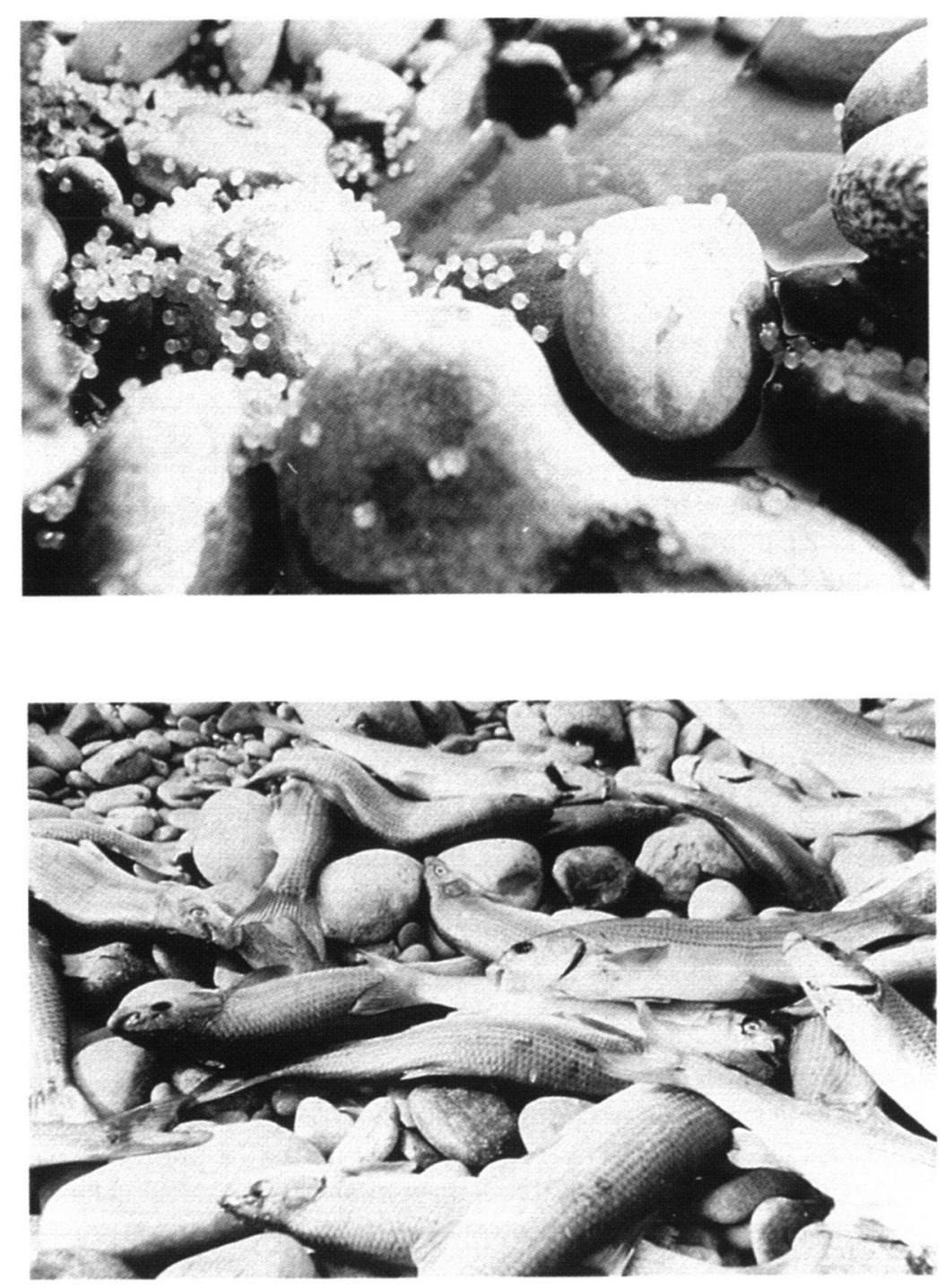

\section{Photos d, e}

Oeufs et reproducteurs mis à sec sur une frayère, après une baisse brutale du débit (Rhône court-circuité de Chautagne).

Photos d, e

Dry spawning and breeding, after a flow failure in the Chautagne by-passed section. 
Tableau II

Biomasses moyennes estimées en $\mathrm{kg} / \mathrm{an}$ (biomasses relatives en \% du total) des captures de Hotu par les pêcheurs professionnels (A) (72\%) et les pêcheurs amateurs (B) (28\%) pour les années 1988 et 1989 (d'après CHANGEUX et ZYLBERBLAT, 1993).

Table II

Estimated average biomass in $\mathrm{kg} /$ year (relative biomass in percentage of the total) of the captures of Nase by professionals (A) (72\%) and amateurs (B) (28\%) for the two years 1988 and 1989 (after CHANGEUX and ZYLBERBLAT, 1993).

\begin{tabular}{|c|c|c|c|c|c|c|}
\hline & $\begin{array}{l}\text { Rhône } \\
\text { amont }\end{array}$ & $\begin{array}{c}\text { Rhône } \\
\text { aval }\end{array}$ & $\begin{array}{l}\text { Rhône } \\
\text { deltaïque }\end{array}$ & $\begin{array}{l}\text { Saône } \\
\text { amont }\end{array}$ & $\begin{array}{c}\text { Saône } \\
\text { aval }\end{array}$ & total \\
\hline A & $\begin{array}{c}8519 \\
(38)\end{array}$ & $\begin{array}{l}1151 \\
(2,5)\end{array}$ & $\begin{array}{r}139 \\
(0,3)\end{array}$ & $\begin{array}{c}61 \\
(2,3)\end{array}$ & $\begin{array}{l}303 \\
(0,4)\end{array}$ & $\begin{array}{c}10174 \\
(5,5)\end{array}$ \\
\hline B & $\begin{array}{c}3420 \\
(24)\end{array}$ & $\begin{array}{c}2410 \\
(16)\end{array}$ & $\begin{array}{l}217 \\
(2)\end{array}$ & $\begin{array}{c}72 \\
(0,6)\end{array}$ & $\begin{array}{c}89 \\
(0,5)\end{array}$ & $\begin{array}{l}6207 \\
(8,7)\end{array}$ \\
\hline \multirow[t]{2}{*}{ total } & $\begin{array}{c}11939 \\
(33)\end{array}$ & $\begin{array}{c}3561 \\
(6)\end{array}$ & $\begin{array}{l}356 \\
(0,7)\end{array}$ & $\begin{array}{c}133 \\
(0,9)\end{array}$ & $\begin{array}{c}392 \\
(0,4)\end{array}$ & $\begin{array}{c}16381 \\
(6,4)\end{array}$ \\
\hline & \multicolumn{3}{|c|}{$\begin{array}{c}15856 \\
(11)\end{array}$} & \multicolumn{2}{|c|}{$\begin{array}{c}525 \\
(0,5)\end{array}$} & \\
\hline
\end{tabular}

par le canal déversoir de Savières, en raison du récent barrage de Chanaz. De plus, le recrutement annuel est difficile dans les affluents du lac, trop irréguliers et pollués (NELVA et al., 1994). La régulation artificielle des débits dans les tronçons court-circuités résiduels du fleuve peut aussi anéantir tout ou partie du recrutement annuel, comme en témoignent les oeufs et les reproducteurs mis brutalement en assec (photos d et e). Pourtant, d'après les statistiques de pêche aux engins dans le Rhône et la Saône (Tab. II) (CHANGEUX et ZYLBERBLAT, 1993), c'est surtout dans le Haut-Rhône que le Hotu se maintient le mieux.

Dans cinq rivières de Moravie (Rép. Tchèque) où le Hotu comptait parmi les espèces les plus abondantes, les populations sont maintenant considérées en danger critique (Fig. 7, LUSK et HALACKA, 1995), avec une baisse de $245 \mathrm{~kg} / \mathrm{ha}$ en 1968 à $12 \mathrm{~kg} / \mathrm{ha}$ en 1990 dans le cas de la Rokytna, où seule intervient une détérioration de la qualité de l'eau. De nombreuses autres rivières sont dans ce cas, en République Tchèque (LUSK, 1995), en Slovénie (POVZ, 1995). Dans la plupart des cours d'eau, ce sont aussi les aménagements qui, seuls ou en plus de la pollution, accélèrent le déclin de l'espèce. Mais dans certaines situations elle arrive parfois à survivre de façon sporadique et même à se reproduire en dépit des fragmentations du cours d'eau et des énormes fluctuations des niveaux d'eau dans les barrages (PROKES et BARUS, 1995). Le déclin démographique des populations de la Meuse et ses affluents, dans lesquels le Hotu a pénétré également au début du siècle par les canaux de navigation, est dû à l'eutrophisation, à la disparition ou à l'altération des habitats et à une surexploitation par la pêche (PHILIPPART, 1994). Depuis une vingtaine d'années, le Hotu est ainsi l'espèce la plus en déclin parmi les Cyprinidés rhéophiles de la zone à Barbeau.

\section{CONCLUSION}

L'introduction spontanée mais d'origine anthropique du Hotu et sa naturalisation presque immédiate dans le réseau hydrographique français peuvent être considérées comme une " réussite " pour l'espèce elle-même, grâce à sa stratégie démographique (forte capacité de reproduction et de croissance) et à sa capacité de développement et d'adaptation (grégarité, migrations, occupation d'une niche alimentaire disponible auparavant peu exploitée). Son expansion biogéographique a donc été rapide et maximale. 


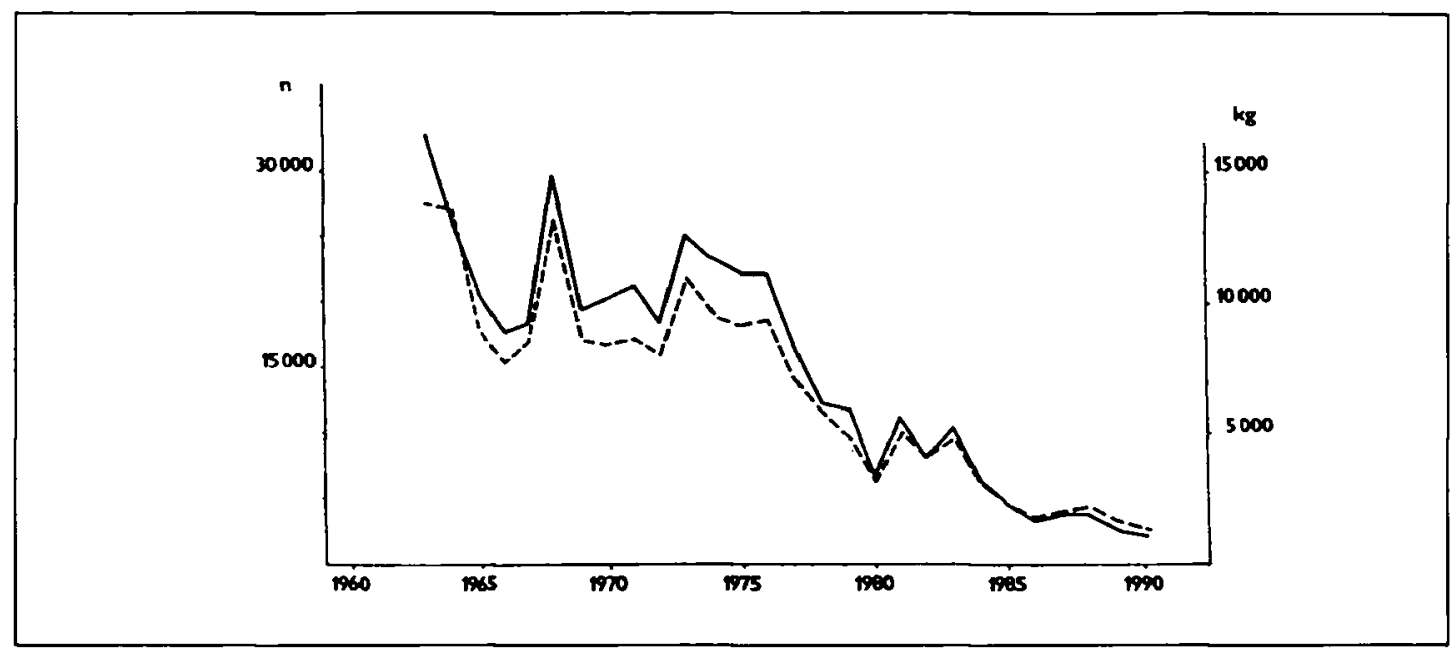

Figure 7

Echantillonnage du Hotu dans la Moravie du sud, de 1963 à 1990 (- nombre d'individus, - - biomasse) (LUSK et HALACKA, 1995).

\section{Figure 7}

Catches of Nase in the Southern Moravian region, for the $1963-1990$ period $1-$ number of individuals, - - - weight) (LUSK and HALACKA, 1995).

Pour le milieu et le peuplement autochtone, la pénétration du Hotu apparaît bénéfique dans la mesure où son arrivée, même massive en certains secteurs de cours d'eau, n'a pas ou peu contraint réellement les espèces autochtones, où elle apporte un stock nutritif abondant pour divers prédateurs, où elle joue un rôle efficace sur le peuplement algal et un rôle épurateur contre une eutrophisation modérée, où elle peut constituer enfin un stock halieutique compensateur.

Cette espèce présente aussi un autre intérêt en tant que descripteur écologique, c'està-dire indicateur d'un type de fonctionnement dans certains cours ou secteurs de cours d'eau des zones à Ombre et à Barbeau. Elle est en effet plus exigeante que chacune de ces deux espèces seules avec lesquelles elle cohabite, d'une part, pour la diversité des habitats nécessaires à la reproduction et à la croissance, d'autre part, pour la nécessité de migrations.

La différenciation d'une zone à Hotu, définie de façon précise par les habitats ponctuels et stationnels, caractérise par exemple mieux le Haut-Rhône avant les aménagements récents et certainement aussi les nouveaux tronçons court-circuités du Rhône. Du point de vue écotoxicologique enfin, une étude est envisagée pour savoir si le Hotu peut servir de bioindicateur ponctuel dans les transferts de substances s'accumulant dans les algues.

La restauration des habitats et d'un minimum de communication entre eux pour assurer les migrations indispensables, la restriction de l'eutrophisation, une régulation moins artificielle des débits et un contrôle de la pêche peuvent simultanément arrêter le déclin du Hotu, qui a déjà fait l'objet de plusieurs réintroductions en Europe centrale et d'une tentative d'introduction en Italie du nord.

\section{BIBLIOGRAPHIE}

CHANGEUX T., ZYLBERBLAT M., 1993. La pêche aux engins sur le domaine public fluvial du bassin du Rhône. Deuxième partie : étude des captures. Bull. Fr. Pêche Piscic., 330, 245-294.

FREYHOF J., 1995. Feeding traces as an indicator of habitat choice in Nase, Chondrostoma nasus. Folia Zoologica, 44 (suppl. 1), 57-61. 
GOLLMANN G., 1995. Biochemical markers in the population genetics of Chondrostoma nasus. Folia Zoologica, 44 (suppl. 1), 83-89.

GYURKO S., 1959. Beitrage zur Ernahrungs biologie der Nase. Arch. Hydrobiol., 56 (1-2), 93101.

KOKUREWICZ B., KOWALEWSKI M., WITKOWSKI A., 1980. Influence of constant and variable temperatures on the embryonic development of European grayling, Thymallus thymallus. Zool. Polon., 27 (3), 335-362.

LIBOVARSKY J., 1977. The fish community in a section of Rokytna Creek after twenty years. Folia Zoologica, 26 (1), 57-60.

LUSK S., 1967. Population dynamics of Chondrostoma nasus (L., 1758) in the Rokytna river. Acta Sc. Nat. Brno, 1, 473-522.

LUSK S., 1995. Influence of valley dams on the changes in fish communities inhabiting streams in the Dyje river drainage area. Folia zoologica, 44 (1), 45-56.

LUSK S., HALACKA K., 1995. Anglers'catches as an indicator of population size of the Nase, Chondrostoma nasus. Folia Zoologica, 44 (2), 185-192.

NELVA A., 1984. Expériences sur la tolérance de Chondrostoma nasus nasus (L., 1758), Hotu (Poisson, Cyprinidé), à une augmentation de la température de l'eau. Rap. int. (non pub.), $10 \mathrm{p}$.

NELVA A., 1985. Biogéographie, démographie et écologie de Chondrostoma nasus nasus (L., 1758) Hotu (Poisson, Téléostéen, Cyprinidé). Thèse Doct. d'Etat, Univ. Lyon I, 365 p.

NELVA A., 1988 a. Origine et biogéographie des deux Chondrostomes français : Chondrostoma nasus et $C$. toxostoma (Pisces, Cyprinidae). Cybium, 12 (4), 287-289.

NELVA A., 1988 b. Profil écologique du Hotu, Chondrostoma nasus (Ostéichtyen, Cyprinidé) dans le Haut-Rhône français: structure d'habitat ponctuel et stationnel. Acta Oecologica, Oecol. Applic., 9 (3), 275-296.

NELVA A., 1989. Structure de population en classes de taille et structure en bancs chez le Hotu, Chondrostoma nasus (Pisces, Cyprinidae), dans le Haut-Rhône français. Cybium, 13 (3), 221-223.

NELVA A., COLLARES-PEREIRA M.J., COELHO M., 1988. Systématique et répartition du genre Chondrostoma Agassiz, 1835 (Pisces, Cyprinidae). Arch. Hydrobiol., 113 (1), 93-112.

NELVA A., PERSAT H., OLIVIER J.M., 1994. One century extension and survival of Chondrostoma nasus populations in the Bourget Lake, French Upper Rhône. Com. 1st International Workshop on Biology of the Nase, Brno (CZ), 17-20 oct. 1994.

PARIS P., 1932. Faune de la Saône moyenne (Cyclostomes et Poissons). Bull. Scient. Bourgogne, II, 47-54.

PENAZ M., LUSK S., 1965. Zu den Ursachen der hohen Sterblichkeit der Naseneier, Chondrostoma nasus L., wahrend der naturlichen Vermehrung. Zool. Listy, 14 (2), 159-170.

PERSAT H., OLIVIER J.M., BOISSON-SANLAVILLE J.M., CHESSEL D., 1994. Long-term changes in the Upper-Rhône population of Chondrostoma nasus facing up to an extensive river development. Com. 1st International Workshop on Biology of the Nase, Brno (CZ), 17-20 oct. 1994.

PHILIPPART J.C., 1977. Contribution à l'hydrobiologie de l'Ourthe. Dynamique des populations et production de 4 espèces de Poissons Cyprinidae : Barbus barbus, Leuciscus cephalus, Chondrostoma nasus, Leuciscus leuciscus. Thèse Univ. Liège, Instit. Zool. Ethol., Aquarium, $237 \mathrm{p}$. 
PHILIPPART J.C., 1994. Geographical distribution and population status of Chondrostoma nasus in the river Meuse catchment area. Com. 1st International Workshop on Biology of the Nase, Brno (CZ), 17-20 oct. 1994.

POVZ M., 1995. Chondrostoma nasus nasus in the waters of Slovenia. Folia Zoologica, 44 (suppl. 1), 9-15.

PROKES M., BARUS V., 1995. Surviving fragments of a Chondrostoma nasus population under extreme conditions in the Mohelno reservoir (Czech Republic). Folia Zoologica, 44 (suppl. 1), 63-67.

SPILLMANN C.J., 1961. Faune de France : 65 poissons d'eau douce. Ed. Paul Chevalier, Paris, $303 \mathrm{p}$.

TUFFERY G., 1969. Etude synoptique de la biologie du Hotu, Chondrostoma nasus (L., 1758). Rap. Stat. Techn. Pêche Piscic., Ecole Nat. Vét. (non pub.). 